UNIVERSIDADE DE SÃO PAULO

INSTITUTO DE GEOCIÊNCIAS

\title{
POTENCIAL FORENSE DE MICROVESTÍGIOS BIOGEOLÓGICOS EM ECOSSISTEMA COSTEIRO DO BRASIL
}

\section{Cynthia Ramos}

Dissertação apresentada ao programa de Geoquímica e Geotectônica para obtenção do título de Mestre em Ciências

Área de Concentração: Geotectônica

Orientador: Prof. Dr. Paulo E. de Oliveira

SÃO PAULO

2019 
Autorizo a reprodução e divulgação total ou parcial deste trabalho, por qualquer meio convencional ou eletrônico, para fins de estudo e pesquisa, desde que citada a fonte.

Serviço de Biblioteca e Documentação do IGc/USP

Ficha catalográfica gerada automaticamente com dados fornecidos pelo(a) autor(a) via programa desenvolvido pela Seção Técnica de Informática do ICMC/USP

Bibliotecários responsáveis pela estrutura de catalogação da publicação:

Sonia Regina Yole Guerra - CRB-8/4208 | Anderson de Santana - CRB$8 / 6658$

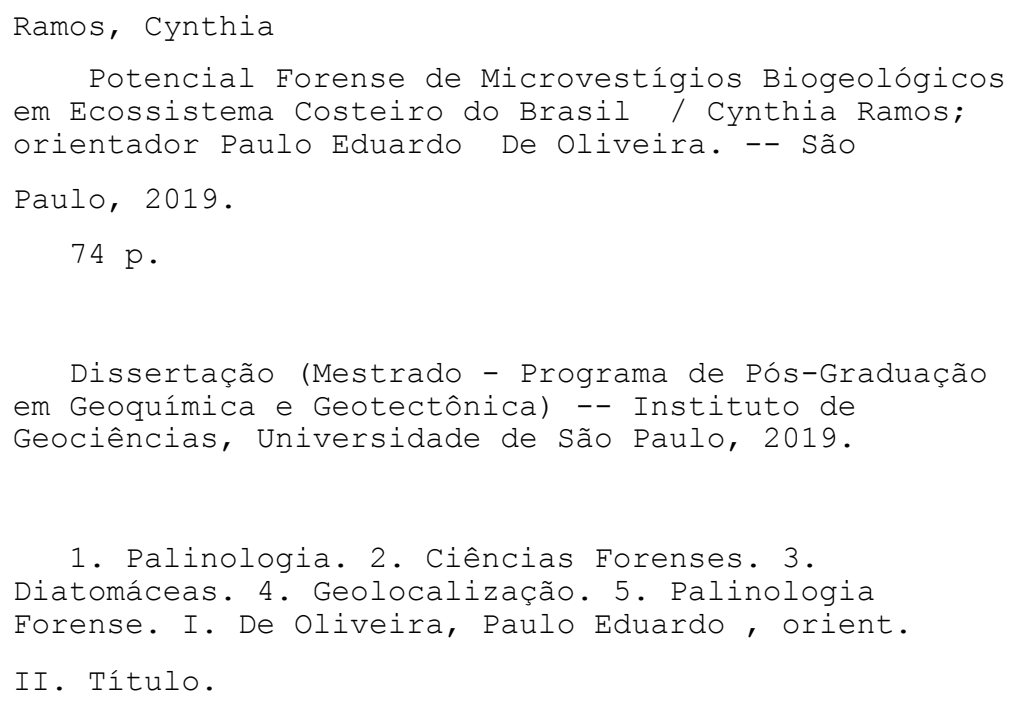


UNIVERSIDADE DE SÃO PAULO

INSTITUTO DE GEOCIÊNCIAS

\title{
POTENCIAL FORENSE DE MICROVESTÍGIOS BIOGEOLÓGICOS EM ECOSSISTEMA COSTEIRO DO BRASIL
}

\section{CYNTHIA RAMOS}

Orientador: Prof. Dr. Paulo Eduardo de Oliveira

Dissertação de Mestrado

№ 835

\author{
COMISSÃO JULGADORA \\ Dr. Paulo Eduardo de Oliveira \\ Dr. Francisco de Assis Ribeiro dos Santos \\ Dr. Fábio Augusto da Silva Salvador
}

SÃO PAULO

2019 


\section{RESUMO}

O termo "Palinologia Forense" refere-se ao uso de grãos de pólen e esporos para fins judiciais, havendo relatos de vários casos ao redor do mundo em que essa técnica se mostrou efetiva. Países como Nova Zelândia, Inglaterra e Estados Unidos utilizam-se amplamente desta técnica e apresentam protocolos consolidados em parceria com instituições acadêmicas para o desenvolvimento e aplicação de metodologias voltadas à essa área. A hipótese de que grãos de pólen e esporos encontrados em peças de roupa de um suspeito ou vítima, possam indicar sua geolocalização no momento do crime, tem sido testada em condições controladas em outros países de vegetação mais homogênea e com padrões de polinização predominantemente anemófila, ou seja, com dispersão através do vento. Embora esta técnica se relacione essencialmente com o estudo de palinomorfos, em sua aplicação ampla, pode também fornecer informações relacionadas a outros microorganismos como foraminíferos e as algas diatomáceas, que normalmente são utilizadas no âmbito forense para a detecção de morte por afogamento. A interpretação dos sinais palinológicos em regiões com vegetação heterogênea são mais difíceis devido à baixa probabilidade de um local apresentar uma assinatura polínica específica e diferente de outros pontos. Esta pesquisa avalia o potencial forense de grãos de pólen, esporos, diatomáceas e foraminíferos como geolocalizadores em uma realidade ambiental como a do Brasil, através do sinal desses microvestígios retido em vestimentas. Foi simulado um trajeto em uma área do litoral paulista, com dois pontos principais de trilha, sendo eles: praia e manguezal, com três indivíduos diferentes. As peças de roupa usadas foram removidas, lavadas e os resíduos foi processado de acordo com as metodologias para extração dos diferentes microvestígios, que foram identificados e contados. A concentração de cada táxon em cada uma das amostras foram analisadas e apresentaram resultados positivos para o contato com área litorânea e em algumas amostras, o contato específico com área de mangue. Essa análise contribui para a criação de protocolos forenses na área de palinologia com o intuito de beneficiar a perícia brasileira.

Palavras-chave: Palinologia, Ciências Forenses, Diatomáceas, Geolocalização, Palinologia Forense. 


\begin{abstract}
The term "Forensic Palynology" refers to the use of pollen grains and spores for judicial purposes, and there have been reports of several cases around the world where this technique has been proved effective. Countries such as New Zealand, England and United States use this technique extensively and present consolidated protocols in partnership with academic institutions for the development and application of methodologies related to this area. The hypothesis that pollen grains and spores found in pieces of clothing of a suspect or victim can indicate their geolocation at the time of the crime has been tested under controlled conditions in other countries with more homogeneous vegetation and with predominantly anemophilic pollination patterns, that is, with dispersion through the wind. Although this technique is essentially related to the study of palynomorphs, in its wide application, it can also provide information related to other microorganisms such as foraminifera and diatom algae, which are normally used in forensic ambit for the detection of death by drowning. The interpretation of the palynological signals in regions with heterogeneous vegetation are more difficult due to the low probability of a site having a specific pollen signature and different from other points. This research evaluates the forensic potential of pollen grains, spores, diatoms and foraminifera as geolocalizers in an environmental reality such as Brazil, through the signal of these microtraces retained in clothing. A route was simulated in an area of the coast of the State of São Paulo, with two main points of track, being: beach and mangrove, with three different individuals. The used garments were removed, washed and the residues were processed according to the methodologies for extraction of the different micro-traces, which were identified and counted. The concentration of each taxon in each of the samples were analyzed and presented positive results for the contact with the coastal area and in some samples, the specific contact with the mangrove area. This analysis contributes to the establishment of forensic protocols in the area of palynology in order to benefit the Brazilian expertise.
\end{abstract}

Key words: Palynology, Forensic Science, Diatoms, Geolocation, Forensic Palynology 


\section{INTRODUÇÃO}

O termo "Palinologia Forense" se refere ao uso judicial da técnica de análise de grãos de pólen e esporos que, devido ao seu tamanho microscópico, podem facilmente aderir a diferentes tipos de superfície. Por essa razão, em uma determinada região podem se tornar uma evidência, ao vincular ou estimar trajetos de uma pessoa ou mais pessoas ou até objetos com a localização onde ocorreu o contato (Bryant et al., 2013).

A Palinologia Forense tem sido aplicada eventualmente como uma ferramenta na resolução de crimes desde a década de 50 , sendo atualmente, uma técnica comumente utilizada em países como Nova Zelândia, Inglaterra e Austrália (Boi, 2018).

Bryant and Mildenhall (1998) citam dezenas de casos ao redor do mundo em que a Palinologia foi usada como parte de investigações policiais, em sua maioria como peça chave para resoluções de crimes, o que demonstra a eficiência da aplicação dessa ciência no âmbito forense.

Embora esta técnica se relacione essencialmente com o estudo de grãos de pólen e esporos, em sua aplicação ampla, pode também fornecer informações relacionadas a outros microorganismos de parede orgânica encontrados em ambientes marinhos ou em águas continentais (Bryant and Mildenhall, 1998). Por exemplo, as diatomáceas, que são algas microscópicas unicelulares formadas por valvas caracterizadas por suas paredes celulares de sílica (Round et al., 1990), que devido a seus requerimentos ecológicos podem facilitar o reconhecimento de ambientes especificos. De uma forma geral, o teste para detectar morte por afogamento é a aplicação mais comum desses organismos na área forense, apesar de nas geociências, o maior interesse por essas algas dentro do âmbito forense seja a identificação da origem de materiais ou indivíduos a partir de locais de investigação. Uma vez que algum objeto tenha entrado em contato com água, seja continental ou litorânea, a análise diatomológica pode descriminar o tipo de ambiente envolvido (Cameron, 2004). Outro exemplo são os foraminíferos, um grupo de microorganismos que até o momento foi pouco empregado em estudos forenses, contudo apresentam um alto poder indicativo de contato com o ambiente litorâneo, podendo ser uma ferramenta útil na perícia (Bowen, 2010). Isso se deve ao fato de que esses protozoários que vivem em habitats marinhos desde a zona costeira, até 
o piso oceânico, difundidos desde os pólos até os trópicos, podendo ser planctônicos ou bentônicos (Armstrong and Brasier, 2005).

Mesmo com a possibilidade de solucionar investigações a aplicação de técnicas como essa ainda é subutilizado pelas polícias cientificas (Amaral, 2018), contudo alguns casos de aplicação de técnicas da Botânica forense mostraram-se altamente eficazes no País (Amaral et al., 2019).

De Oliveira e Suguio (2005) evidenciaram a importância do desenvolvimento de estudos forenses voltados para o emprego de técnicas de análises palinológicas e diatomológicas de sedimentos. Tendo como exemplo um furto de containers com equipamentos eletrônicos transportados do Porto de Santos, SP até a cidade São Paulo, onde os equipamentos foram substituídos por sacos de areia. As análises desses sedimentos e outros materiais argilosos revelaram a presença de grãos de pólen típicos da Mata Atlântica e de manguezais da Baixada Santista, além de diatomáceas típicas da região costeira do estado de São Paulo. Essas evidências levaram à conclusão de que o furto havia ocorrido nas redondezas do Porto e não no Município de São Paulo.

É sabido que o tipo de solo e a alta diversidade da vegetação de uma área podem dificultar a identificação de locais específicos (Wiltshire, 2016), principalmente em um país como o Brasil, que apresenta grande heterogeneidade na vegetação, inclusive dentro de um mesmo ecossistema (Garcia e Pirani, 2005; Magnago et al., 2011; Assis et al., 2011), o que aumenta a complexidade de interpretação do sinal polínico. Portanto, apesar de atualmente existirem reconhecidos protocolos palinológicos forenses internacionais (Mildenhall et al., 2006; Wiltshire, 2016), se faz necessária a criação de metodologias adaptadas à realidade ambiental brasileira. Dessa forma, para promover o intercâmbio de conhecimentos forenses na comunidade acadêmica brasileira e estimular 0 estabelecimento de parcerias com órgãos de perícia criminal, a Coordenação de Aperfeiçoamento de Pessoal de Nível Superior (CAPES) criou o Programa PróForense em 2014, através do edital Pró-Forense. Com esse estímulo, as ciências forenses passaram a fazer parte das atividades de pesquisa em instituições acadêmicas de forma mais sistemática e estruturada. Essa prática de parcerias com instituições de pesquisa já é comumente aplicada em países como Estados Unidos, Inglaterra e Nova Zelândia, com protocolos mais consolidados (Community Committee on Identifying the Needs of the Forensic Sciences, 2009). 
Como parte integrante de projeto inserido neste programa da CAPES, este trabalho tem como objetivo estabelecer procedimentos de coleta e análise de interesse forense levando em conta a presença de grãos de pólen, entre outros microvestígios em vestimentas. Testou-se a possibilidade de gerar dados sobre a transferência de vestígios do ambiente para essas peças e avaliar o grau de associação com o ecossistema que o material entrou em contato.

O princípio de Edmond Locard, um dos pioneiros em Ciências Forenses (Locard, 1920, 1929) que postula "todo contato deixa uma marca" é, neste trabalho, associado ao fato de que espécies vegetais possuem requerimentos ecológicos específicos (Good, 1964), por vezes associadas à áreas de endemismo, ou seja, com ocorrência exclusiva em uma região particular (Carvalho, 2009b), permitindo a identificação desse contato.

Isto porque, os grãos de pólen possuem estruturas que facilitam sua dispersão através de diferentes vetores. Na vegetação neotropical, onde predomina a polinização zoófila, ou seja, por animais, (Faegri e van der Pijl, 1979; Ramalho e Batista, 2005), a parede externa (exina) dos grãos, apresenta ornamentações como, por exemplo, espinhos, clavas, ou até mesmo por pollenkitt, uma substância aderente que permite o transporte do grão por insetos e animais que entrem em contato com a estrutura de reprodução masculina da flor (Traverse, 2007). Portanto, os tecidos de vestuários podem ocasionalmente ser vetores análogos.

Desta forma, prevê-se encontrar associações de tipos polínicos em resíduos obtidos de peças de roupas que foram expostas a áreas de vegetação específica de uma região litorânea do estado de São Paulo. Espera-se que essa assinatura polínica combinada com o sinal de táxons de diatomáceas marinhas e continentais possa indicar a geolocalização do contato.

Estudos forenses em ambientes costeiros do Brasil são de grande importância devido à localização de grandes centros urbanos e atividades portuárias, possíveis cenários de diferentes tipos de crimes (Eski, 2011). Um exemplo é a Região Metropolitana da Baixada Santista, que mantém desde os anos 80 o segundo lugar no Estado de São Paulo em questão de mortes violentas (Camargo, 2007). 


\section{CONCLUSÕES E SUBSÍDIOS A FUTUROS PROTOCOLOS FORENSES}

Esta pesquisa exploratória dentro da estratégia desenvolvida pela CAPES para impulsionar as ciências forenses no Brasil, ancorada por vínculos acadêmicos e periciais, apresentou resultados positivos para subsidiar protocolos forenses na área de palinologia no país.

O trabalho trouxe uma temática pioneira no Brasil, simulando rotas para checagem da retenção diferencial de microvestígios em função de tipos de vestimenta, associando diferentes fontes de evidência, como palinomorfos, diatomáceas e foraminíferos. Tudo isso com o desafio de lidar com a realidade ambiental do país que apresenta uma vegetação com padrão de polinização predominantemente zoófila e extremamente heterogênea.

Observou-se o fato de que sinais específicos são retidos simultaneamente em diferentes materiais e peças, informação de grande utilidade na rotina pericial, uma vez que esse conhecimento garante uma maior disponibilidade de amostras do mesmo indivíduo.

Desconsiderar os táxons ocorrentes apenas uma vez, manteve uma análise conservadora, o que concorda com a presunção de inocência, uma garantia processual do direito brasileiro que beneficia o acusado no caso de dúvida razoável quanto à sua culpabilidade.

Os resultados também mostraram a necessidade de desenvolvimento de pesquisas adicionais que agreguem mais robustez à interpretação estatística dos resultados tais como amostras de solo para controle e repetições de trajetos. Isso porque a complexidade da identificação de palinomorfos em ecossistemas como a Mata Atlântica do litoral do estado de São Paulo, em que raramente é possível apontar uma espécie endêmica, dificulta o direcionamento para a exatidão geográfica.

Portanto, mesmo com importantes resultados para a interpretação forense em área litorânea da região Sudeste do Brasil - onde se localiza o maior e o mais importante porto da América Latina - é recomendada a repetição deste estudo em outras áreas do país. Sua aplicação em outros ecossistemas e em diferentes épocas do ano irá expandir e corroborar com a elaboração de protocolos palinológicos para a perícia cientifica brasileira. 


\section{REFERÊNCIAS BIBLIOGRÁFICAS}

Algaebase, 2019, Algaebase:,

http://www.algaebase.org/search/?species=genusname speciesname (accessed January 2019).

Amaral, M.M., 2018, Botânica e Palinologia Forenses, in Dias Filho, C.R. e Francez, P.A.C. eds., Introdução à Biologia Forense, São Paulo, Millennium, p. 157-211.

Amaral, P.G.C., 2003, Contribuição palinológica ao estudo da evolução do manguezal do rio Itanhaém, litoral sul de São Paulo: Universidade de São Paulo, $71 \mathrm{p}$.

Amaral, M.M., Bicudo, C.E.M., Magalhães e Silva, F.H., De Oliveira, P.E., e Angyalossy, V., 2019, A Botânica vai ao tribunal (M. M. Amaral, Ed.): São Paulo, RiMa, $72 \mathrm{p}$.

Araújo, D.S.D., e Lacerda, L.D., 1987, A natureza das restingas: Ciência Hoje, v. 6, p. 42-48.

Armstrong, H., e Brasier, M., 2005, Microfossils: Oxford, Blackwell Publishing, 296 p. Assis, M.A. et al., 2011, Florestas de restinga e de terras baixas na planície costeira do sudeste do Brasil: vegetação e heterogeneidade ambiental: Biota Neotropica, v. 11, p. 103-121, doi:10.1590/S1676-06032011000200012.

Barros, F., Melo, M.M.R.F., Chiea, S.A.C., Wanderley, M.G.L., e Jung-Mendaçolli, S.L., 1991, Flora Fanerogâmica da llha do Cardoso - Caracterização geral da vegetação e listagem das espécies ocorrentes: São Paulo, Hucitec, 184 p.

Battarbee, R.W., 1986, Diatom Analysis, in Berglund, B.E. ed., Handbook of Holocene Palaeoecology and Palaeohydrology, Wiley, p. 527-570.

Boi, M., 2018, The Importance of Palynology in Forensic Investigations: Forensic Science \& Addiction Research, v. 3, p. 2-3, doi:10.31031/FSAR.2018.03.000580.

Boltovskoy, E., e Wright, R., 1976, Recent Foraminifera: Dordrecht, Springer Netherlands, 575 p., doi:10.1007/978-94-017-2860-7.

Bowen, A.M., 2010, Forensic applications of Foraminifera: The Microscope, v. 58, p. $3-18$,

http://www.csa.com/htbin/dbrng.cgi?username=usfish\&access=usfish $1590 \& \mathrm{db}=$ georef-set-c\&qry=an\%3D2010-088656\&adv=1\&fed=results.

Bryant, V.M., Bush, M.B., e Siska, P., 2013, Forensic Geolocation Based on Pollen 
Analysis, in Hwang, G.M. and Masters, D. eds., Pollen Coalition Workshop, McLean, VA, AASP - The Palynological Society, p. 10-20.

Bryant, V.M., e Mildenhall, D.C., 1998, Forensic palynology: a new way to catch crooks, in Bryant, V.M. e Wrenn, J.W. eds., New developments in palynomorph sampling, extraction and analysis, American Association of Stratigraphic Palynologists Foundation, p. 145-155, http://anthropologyworldnews.tamu.edu/faculty/bryant/publications/BryantMildenhall-1998-New-Way-to-Catch-Crooks.pdf.

Buzato, S., Sazima, M., e Sazima, I., 1994, Pollination of three species of Abutilon (Malvaceae) intermediate between bat and hummingbird flower syndromes: Flora, v. 189, p. 327-334, doi:10.1016/S0367-2530(17)30613-8.

Camargo, A.B.M., 2007, Mortes por Causas Violentas no Estado de São Paulo - a influência das agressões: São Paulo em Perspectiva, v. 21, p. 31-45.

Cameron, N.G., 2004, The use of diatom analysis in forensic geoscience, in Pye, K. e Croft, D.J. eds., Forensic Geoscience: Principles, Techniques and Applications, London, The Geological Society of London, v. 232, p. 277-280, doi:10.1144/gsl.sp.2004.232.01.25.

Carvalho, A.M.C., 2009a, Guilda de abelhas e outros visitantes de Matayba guianensis (Sapindaceae) em vegetação de Cerrado: Universidade Federal de Uberlândia, $147 \mathrm{p}$.

Carvalho, C.J.B., 2009b, Padrões de endemismos e a conservação da biodiversidade, in Silva, J.M.C. ed., Megadiversidade - Os desafios científicos para a conservação da biodiversidade no Brasil, Belo Horizonte - MG', Conservação Internacional, v. 5, p. 77-86.

Cerqueira, R., 2000, Biogeografia das Restingas, in Esteves, F.A. e Lacerda, L.D. eds., Ecologia de Restingas e Lagoas Costeiras, Macaé, RJ, NUPEM / UFRJ, p. 65-75.

Colinvaux, P., De Oliveira, P.E., e Patiño, J.E.M.., 1999, Amazon Pollen Manual and Atlas: CRC Press, $332 \mathrm{p}$.

Community Committee on Identifying the Needs of the Forensic Sciences, 2009, Strenghening Forensic Science in the United States: A Path Forward. National Research Council: 352 p., doi:10.1016/0379-0738(86)90074-5.

Couto, O.S., e Cordeiro, R.M.S., 2005, Manual de reconhecimento de espécies vegetais da restinga do estado de São Paulo: São Paulo, Secretaria do Meio 
Ambiente, $440 \mathrm{p}$.

Dorneles, L.L., Zillikens, A., e Padilha, M.T.S., 2013, Biologia da polinização de Euterpe edulis Martius (Arecaceae) e associação com abelhas sociais (Apidae: Apini) em sistema agroflorestal na llha de Santa Catarina: Iheringia, v. 68, p. 47-57.

van Dulmen, A., 2001, Pollination and phenology of flowers in the canopy of two contrasting rain forest types in Amazonia, Colombia: Plant Ecology, v. 153, p. 73-85.

Eichler, P.P.B., Eichler, B.B., David, C.J., Miranda, L.B., e Sousa, E.C.P.M., 2006, The estuary ecosystem of Bertioga, São Paulo, Brasil: Journal of Coastal Research, p. 1110-1113.

Erdtman, G., 1986, Pollen Morphology and Plant Taxonomy: Leiden, E. J. Brill, 539 p.

Eski, Y., 2011, "Port of call": Towards a criminology of port security: Criminology and Criminal Justice, v. 11, p. 415-431, doi:10.1177/1748895811414593.

Faegri, K., e van der Pijl, L., 1979, The principles of pollination ecology: Oxford, Pergamon Press Ltd., $244 \mathrm{p}$.

Ferri, M.G., 1980, Vegetação Brasileira: Belo Horizonte / São Paulo, Itatiaia / Editora da Universidade de São Paulo, 157 p.

Fierz, M.S.M., e Rosa, F.S., 1999, A paisagem x evolução do uso e ocupação do solo em Bertioga, litoral paulista: Revista do Departamento de Geografia1, v. 13, p. 259-287.

Forzza, R.C. et al., 2010, Catálogo de Plantas e Fungos do Brasil: Rio de Janeiro, Andrea Jakobsson Estúdi / Jardim Botânico do Rio de Janeiro, 872 p.

Fundação Florestal, 2018, Parque Estadual Restinga de Bertioga - Plano de Manejo:, arquivos.ambiente.sp.gov.br/.../2019/01/plano-de-manejo-pe-restingade-bertioga.pdf.

Garcia, R.J.F., e Pirani, J.R., 2005, Análise florística, ecológica e fitogeográfica do Núcleo Curucutu, Parque Estadual da Serra do Mar (São Paulo, SP), com ênfase nos campos juntos à crista da Serra do Mar: Hoehnea, v. 32, p. 1-48.

Gomes, R., Pinheiro, M.C.B., Lima, H.A., e Santiago-Fernandes, L.D.R., 2010,

Biologia fl oral de Manilkara subsericea e de Sideroxylon obtusifolium

(Sapotaceae) em restinga: Revista Brasil. Bot., v. 33, p. 271-283.

Good, R., 1964, The Geography of Flowering Plants: London, Logman Group, 518 p. 
Gottsberger, G., e Silberbauer-Gottsberger, I., 2018, How are pollination and seed dispersal modes in Cerrado related to stratification? Trends in a cerrado sensu stricto woodland in southeastern Brazil, and a comparison with Neotropical forests: Acta Botanica Brasilica, v. 32, p. 434-445, doi:10.1590/0102$33062018 \mathrm{abb} 0186$.

Grimm, E.C., e Troostheide, C.D., 1994, Tilia 2.0 - program for plotting palynological diagrams:

Guedes, D., Barbosa, L.M., e Martins, S.E., 2006, Composição florística e estrutura fitossociológica de dois fragmentos de floresta de restinga no Município de Bertioga, SP, Brasil: Acta Botanica Brasilica, v. 20, p. 299-311, doi:10.1590/S0102-33062006000200006.

Guedes, M.L.S., Batista, M.A., Ramalho, M., Freitas, H.M.B., e Silva, E.M., 2005, Breve incursão sobre a biodiversidade da Mata Atlântica, in Franke, C.R., Rocha, P.L.B., Klein, W., e Gomes, S.L. eds., Mata Atlântica e Biodiversidade, Salvador, Edufba, p. 461.

Hammer, Ø., Harper, D.A.T., e Ryan, P.D., 2001, PAST: Paleontological statistics software package for education and data analysis: Palaeontologia electronica, $v$. 4, p. 9.

Hill, M.O., e Gauch Jr., H.G., 1980, Detrended Correspondence Analysis: An Improved Ordination Technique: Vegetatio, v. 42, p. 47-58.

IBGE, 2019, Instituto Brasileiro de Geografia e Estatística:, https://cidades.ibge.gov.br/brasil/sp/bertioga/panorama (accessed June 2019).

INMET, 2019, Instituto Nacional de Meteorologia:, http://www.inmet.gov.br/portal/index.php?r=estacoes/estacoesAutomaticas (accessed March 2019).

Kojima, R.K., Kameyama, C., e Magenta, M.A.G., 2019, Acanthaceae Juss. no Parque Estadual Restinga de Bertioga, Estado de São Paulo, Brasil: Hoehnea, v. 46, p. 1-16, doi:10.1590/2236-8906-46/2018.

Krammer, K., e Lange-Bertalot, H., 1986, Bacillarophyceae, 1. Teil: Naviculaceae: Gustav Fischer, 876 p.

Langenheim, J.H., Hackner, B.L., e Barlett, A., 1967, Mangrove pollen at the depositional site of oligo-miocene amber from Chiapas, Mexico: Harvard University Herbaria, v. 21, p. 289-324, http://data.gbif.org/datasets/resource/1827. 
Ledru, M., Salatino, M.L.F., Ceccantini, G., Salatino, A., Pinheiro, F., e Pintaud, J., 2007, Regional assessment of the impact of climatic change on the distribution of a tropical conifer in the lowlands of South America: Diversity and Distributions, v. 13, p. 761-771, doi:10.1111/j.1472-4642.2007.00389.x.

Lehmkuhl, E.A., 2009, Diatomáceas (Diatomeae) da Baía de Guaratuba, estado do Paraná, Brasil: Universidade Federal do Paraná, 282 p.

Lenza, E., e Oliveira, P.E., 2005, Biologia reprodutiva de Tapirira guianensis Aubl. (Anacardiaceae), uma espécie dióica em mata de galeria do Triângulo Mineiro, Brasil: Revista Brasileira de Botânica, v. 28, p. 179-190, doi:10.1590/s010084042005000100015.

Levin, E.A., Morgan, R.M., Scott, K.R., e Jones, V.J., 2017, The transfer of diatoms from freshwater to footwear materials: An experimental study assessing transfer, persistence, and extraction methods for forensic reconstruction: Science \& Justice, v. 57, p. 349-360, doi:10.1016/j.scijus.2017.05.005.

Lewis, W.H., 1986, Airborne Pollen of the Neotropics: Grana, v. 25, p. 75-83, doi:10.1080/00173138609429936.

Locard, E., 1929, L'analyse des poussieres en criminalistique: Revue Internationale de Criminalistique, v. 1, p. 176-249.

Locard, E., 1920, L'enquete criminelle et les methodes scientifiques: Paris, Flammarion, $303 \mathrm{p}$.

Lorente, F.L., Buso Jr., A.A., De Oliveira, P.E., e Pessenda, L.C.R., 2017, Atlas Palinológico: Laboratório 14C - Cena/USP: Piracicaba, FEALQ, 333 p.

Magalhães e Silva, F.H., e Santos, F.A.R., 2009, Pollen morphology of the shrub and arboreal flora of mangroves of Northeastern Brazil: Wetlands Ecology and Management, v. 17, p. 423-443, doi:10.1007/s11273-008-9118-2.

Magnago, L.F.S., Martins, S. V., e Pereira, O.B., 2011, Heterogeneidade florística das fitocenoses de restingas nos estados do Rio de Janeiro e Espírito Santo, Brasil: Revista Árvore, v. 35, p. 245-254, doi:10.1590/s010067622011000200009.

Malerbo-Souza, D.T., Nogueira-Couto, R.H., e Couto, L.A., 2004, Honey Bee Attractants and Pollination in sweet orange, Citrus sinenensis (L.) Osbeck, var. Pera Rio: J. Venom. Anim. Toxins incl. Trop. Dis., v. 10, p. 144-153. Martins, S.E., Rossi, L., Sampaio, P. de S.P., e Magenta, M.A.G., 2008, Caracterização florística de comunidades vegetais de restinga em Bertioga, SP, 
Brasil: Acta Botanica Brasilica, v. 22, p. 249-274, doi:10.1590/S010233062008000100024.

Menezes, M.P.M., Oliveira, D., e Mello, C.F., 1997, Pollination of red mangrove, Rhizophora mangle, in northern Brazil: Acta Hort, v. 437, p. 431-434.

Metzeltin, D., e Lange-Bertalot, H., 1998, Tropische Diatomeen in Sudamerika, I Tropical diatoms of South America: Konigstein, Koeltz Scientific Books, 695 p.

Mildenhall, D.C., Wiltshire, P.E.J., e Bryant, V.M., 2006, Forensic palynology: Why do it and how it works: Forensic Science International, v. 163, p. 163-172, doi:10.1016/j.forsciint.2006.07.012.

Morgan, R.M., 2017, Conceptualising forensic science and forensic reconstruction.

Part I: A conceptual model: Science \& Justice, v. 57, p. 455-459, doi:10.1016/j.scijus.2017.06.002.

Morgan, R.M., Freudiger-Bonzon, J., Nichols, K.H., Jellis, T., Dunkerley, S., Zelazowski, P., e Bull, P.A., 2009, The Forensic Analysis of Sediments Recovered from Footwear, in Ritz, K., Dawson, L., e Miller, D. eds., Criminal and Environmental Soil Forensics, Dordrecht, Springer Netherlands, p. 253-269, doi:10.1007/978-1-4020-9204-6_16.

Moro, R.S., e Fürstenberger, C.B., 1997, Catálogo dos principais parâmetros ecológicos de diatomáceas não-marinhas: Ponta Grossa, PR, Editora da UEPG, $282 \mathrm{p}$.

Muller, J., 1959, Palynology of Recent Orinoco delta and shelf sediments : Reports of the Orinoco shelf: Micropaleontology, v. 5, p. 1-32.

Negrão, E.N.M., Santos, T.F., Pantoja, M. V, e Maués, M.M., 2013, Características sobre a biologia da polinização e dispersão de sementes de espécies prioritárias para o manejo florestal no estado do Pará, in $64^{\circ}$ Congresso Nacional de Botânica, Belo Horizonte, MG, http://ainfo.cnptia.embrapa.br/digital/bitstream/item/99176/1/resumo-ins20596id5054.pdf.

Negrini Neto, O., 2009, Manual de Procedimentos Básicos de Laboratório do Centro de Exames, Análises e Pesquisas.:

De Oliveira, P.E., e Suguio, K., 2005, Estudos quaternários e sua aplicação forense: caso estudo de containers furtados no estado de São Paulo, in XI Congresso da Associação Brasileira de Estudos Quaternários, Guarapari, ES, ABEQUA, p. 15. 
Opler, P.A., Frankiet, G.W., e Baker, H.G., 1980, Comparative Phenological Studies of Treelet and Shrub Species in Tropical Wet and Dry Forests in the Lowlands of Costa Rica: British Ecological Society, v. 68, p. 167-188.

Patrick, R., e Reimer, C.W., 1975, The diatoms of the United State: Philadelphia, Acad. nat. Sci. Philad., 213 p.

Patrick, R., e Reimer, C.W., 1966, The diatoms of the United States: Philadelphia, Acad. nat. Sci. Philad., 688 p.

Pinto Sobrinho, F.A., Souza, C.R.G., e Mogollón, J.E.J.B., 2011, Análise estrutural de florestas de restinga associadas a depósitos marinhos pleistocênicos e holocênicos na Bacia do Rio Itaguaré, Bertioga (SP): Revista do Instituto Geológico, v. 32, p. 27-40.

Ramalho, M., e Batista, M.A., 2005, Polinização na Mata Atlântica: perspectiva ecológica da fragmentação, in Franke, C.R., Rocha, P.L.B., Klein, W., e Gomes, S.L. eds., Mata Atlântica e Biodiversidade, Salvador, Edufba, p. 93-142.

Raven, P.H., Evert, R.F., e Eichhorn, S.E., 1992, Plantas Vasculares sem sementes, in Biologia Vegetal, New York, Worth Publishiers, Inc., p. 294-332.

Riding, J.B., Rawlins, B.G., e Coley, K.H., 2007, Changes in soil pollen assemblages on footwear worn at different sites: Palynology, v. 31, p. 135-151, doi:10.1080/01916122.2007.9989640.

Rizzini, C.T., 1979, Tratado de Fitogeografia do Brasil - aspectos sociológicos e florísticos: São Paulo, HUCITEC LTDA, 374 p.

Rodrigues, A.R., Eichler, P.P.B., e Eichler, B.B., 2003, Utilização de foraminíferos no monitoramente do canal de Bertioga (SP, Brasil): Atlântica, Rio Grande, v. 25, p. 35-51.

Roubik, D.W., e Patiño, J.E.M., 1991, Pollen and Spores of Barro Colorado Island: Missouri Botanical Garden, 268 p.

Round, F.E., Crawford, R.M., and Mann, D.G., 1990, The diatoms: biology \& morphology of the genera: Cambridge, Cambridge University Press, $747 \mathrm{p}$.

Salgado-Laboriau, M.L., 2007, Critérios e Técnicas para o Quaternário: São Paulo, Edgard Blücher, 387 p.

Sampaio, D., Souza, V.C., Oliveira, A.A., Paula-Souza, J., e Rodrigues, R.R., 2005, Árvores da Restinga: São Paulo, Neotrópica, 277 p.

Santos, M.G., Sylvestre, L.S., e Araújo, D.S.D., 2004, Análise florística das pteridófitas do Parque Nacional da Restinga de Jurubatiba, Rio de Janeiro, 
Brasil: Acta bot. bras., v. 18, p. 271-280.

Schaeffer-Novelli, Y., 2018a, A diversidade do ecossistema manguezal, in Fumi, M. ed., Atlas dos Manguezais do Brasil, Brasília, Instituto Chico Mendes de Conservação da Biodiversidade, p. 21-34.

Schaeffer-Novelli, Y., 2018b, Mangue e Manguezal, in Fumi, M. ed., Atlas dos Manguezais do Brasil, Brasíla, Instituto Chico Mendes de Conservação da Biodiversidade, p. 15-20, http://www.icmbio.gov.br/portal/images/stories/manguezais/atlas_dos_manguez ais_do_brasil.pdf.

Schmiegelow, J.M.M., Gianesella, S.M.F., Simonetti, C., Saldanha-Corrêa, F.M.P., Feoli, E., Santos, J.A.P., Santos, M.P., Ribeiro, R.B., e Sampaio, A.F.P., 2008, Primary producers in Santos estuarine system, in Neves, R., Baretta, J., e Mateus, M. eds., Perspectives on integrated coastal zone management in South America, Lisboa, Instituto Superior Técnico, p. 161-174.

Scott, K.R., Morgan, R.M., Jones, V.J., e Cameron, N.G., 2014, The transferability of diatoms to clothing and the methods appropriate for their collection and analysis in forensic geoscience: Forensic Science International, v. 241, p. 127-137, doi:10.1016/j.forsciint.2014.05.011.

Silva, R.M., Mehlig, U., Santos, J.U.M., e Menezes, M.P.M., 2010, The coastal restinga vegetation of Pará, Brazilian Amazon: a synthesis: Revista Brasil. Bot., v. 33 , p. $563-573$.

Silva, M.F.F., Secco, R.S., e Lobo, M.G., 1996, Aspectos Ecológicos da vegetação rupestre da Serra dos Carajás, Estado do Pará, Brasil: Acta Amazônica, v. 26, p. $17-44$.

Souza, C.R.G., 2007, Ambientes Sedimentares de Planície Costeira e Baixa-Média Encosta em Bertioga (SP), in XI Congresso da Associação Brasileira de Estudos Quaternários, Belém, PA, ABEQUA, p. 1-4.

Souza, C.R.G., Bendazoli, A., Sugiyama, M., Lopes, E.A., e Kirizawa, M., 1997, A relação entre o meio físico e a biota no estudo da "restinga" do estado de São Paulo, in Angulo, R.J. ed., VI Congresso da Associação Brasileira de Estudos do Quaternário, Paraná, ABEQUA, p. 367-372.

Souza, C.R.G., Hiruma, S.T., Sallun, A.E.M., Ribeiro, R.R., e Sobrinho, J.M.A., 2008, "Restinga" Conceitos e Empregos do Termo no Brasil e Implicações na Legislação Ambiental: São Paulo, Instituto Geológico, 104 p. 
Stockmarr, J., 1971, Tablets with spores used in absolute pollen analysis: Pollen et Spores, v. 13, p. 615-621.

Suhs, R.B., Somavilla, A., Köhler, A., e Putzke, J., 2009, Vespídeos (Hymenoptera, Vespidae) vetores de pólen de Schinus terebinthifolius Raddi (Anacardiaceae), Santa Cruz do Sul, RS, Brasil: Revista Brasileira de Biociências, v. 7, p. 138143, http://www.ufrgs.br/seerbio/ojs/index.php/rbb/article/view/1123.

Taura, H.M., e Laroca, S., 2004, Biologia da Polinização: interações entre as abelhas (Hym., Apoidea) e as flores de Vassobia breviflora (Solanaceae): Acta Biológica Paranaense, v. 33, p. 143-162, doi:10.5380/abpr.v33i0.630.

Tavares, A.C.M., 2011, Atributos e visitantes florais e polinização em uma área de Floresta Ombrófila Densa Submontana do litoral norte de São Paulo:

Universidade Estadual de Campinas, $71 \mathrm{p}$.

Maroja, T. E., Silva, M. A. C., Andrade L. K. F., e Quirino, Z. G. M., 2018, Pollination and dispersal syndromes of herbaceous species in urban green areas in Joao Pessoa, Paraiba.: Revista Brasileira de Meio Ambiente, v. 4, p. 69-84.

Todzia, C.A., 1988, Chlorantaceae: Hedyosmum: New York, New York Botanical Garden Press for Flora Neotropica, v. 48, 1-138 p.

Traverse, A., 2007, Paleopalynology: London, Springer Science \& Business Media, $813 \mathrm{p}$.

Trigo, M.D.M., Cabezudo, B., Recio, M., e Toro, F.J., 1996, Annual, daily and dirunal variations of Urticaceae airborne pollen in Málaga (Spain): Aerobiologia, v. 12, p. 85-90, doi:10.1007/BF02446599.

Veloso, H.L., Rangel-Filho, A.L.R., e Lima, J.C.A., 1991, Classificação da vegetação brasileira, adaptada a um sistema universal: Rio de Janeiro, IBGE, 124 p.

Vieira, C.E.L., 2011, Diatomáceas, in Carvalho, I.S. ed., Paleontologia - Microfósseis paleoinvertebrados, Rio de Janeiro, Interciência, p. 554.

Villwock, J.A., Lessa, G.C., Suguio, K., Angulo, R.J., e Dillenburg, S.R., 2005, Geologia e Geomorfologia de Regiões Costeiras, in Souza, C.R.G., Suguio, K., Oliveira, A.M.S., e De Oliveira, P.E. eds., Quaternário do Brasil, Ribeirão Preto, Holos, p. 382.

Webb, J.C., Brown, H.A., Toms, H., e Goodenough, A.E., 2018, Differential retention of pollen grains on clothing and the effectiveness of laboratory retrieval methods in forensic settings: Forensic Science International, v. 288, p. 36-45, doi:10.1016/j.forsciint.2018.04.010. 
Wetzel, C.E., 2011, Biodiversidade e distribuição de diatomáceas (Ochrophyta, Bacillariophyceae) na bacia hidrográfica do Rio Negro, Amazonas, Brasil: Instituto de Botânica, 1911 p.

Wiltshire, P.E.J., 2016, Protocols for forensic palynology: Palynology, v. 40, p. 4-24, doi:10.1080/01916122.2015.1091138.

Zamora, N., Medeanic, S., e Corrêa, I.C.S., 2007, Microforaminíferos como Indicadores Paleoambientais : Estudos Palinológicos da Região Sul da Costa Rica, América Central: Gravel, v. 5, p. 75-87.

Zappi, D.C. et al., 2015, Growing knowledge: an overview of Seed Plant diversity in Brazil: Rodriguésia, v. 66, p. 1085-1113, doi:10.1590/2175-7860201566411.

Zavada, M.S., McGraw, S.M., e Miller, M.A., 2007, The role of clothing fabrics as passive pollen collectors in the north-eastern United States: Grana, v. 46, p. 285-291, doi:10.1080/00173130701780104.

Zündt, C., 2006, Baixada Santista: uso, expansão e ocupação do solo, estruturação de rede urbana regional e metropolização, in Cunha, J.M.P. ed., Novas metrópoles paulistas: população, vulnerabilidade e segregação, Campinas, Núcleo de Estudos de População, Universidade Estadual de Campinas, p. 305336. 